\title{
Ville-nature et architectures des milieux
}

Urban Nature and the Architecture of Milieus

Frédéric Bonnet, Stéphane Bonzani et Chris Younès

\section{OpenEdition}

Journals

Édition électronique

URL : http://journals.openedition.org/crau/574

DOI : $10.4000 /$ crau. 574

ISSN : 2547-5746

Éditeur

Éditions du patrimoine

Édition imprimée

Date de publication : 1 novembre 2012

Pagination : 182-191

ISBN : 978-2-7577-0108-9

ISSN : $1296-4077$

Référence électronique

Frédéric Bonnet, Stéphane Bonzani et Chris Younès, «Ville-nature et architectures des milieux », Les Cahiers de la recherche architecturale et urbaine [En ligne], 26/27 | 2012, mis en ligne le 01 novembre 2017, consulté le 03 mai 2019. URL : http://journals.openedition.org/crau/574 ; DOI : 10.4000/ crau. 574 
II s'agit d'interroger les liaisons entre ville-naturemonde et architecture des milieux, en se centrant sur les questions d'habiter, de lieux et d'artefact.

\section{Le milieu ne va pas sans} multiplicité et re-création ; il y en a toujours plusieurs en jeu (environnemental, social, technique, culturel, psychologique...), qui se superposent, s'entremêlent, se rencontrent, se métamorphosent.

\section{Ville-nature et architectures des milieux}

FRÉDÉRIC BONNET

STÉPHANE BONZANI

CHRIS YOUNÈS

Depuis plusieurs années, nous développons une problématique portant sur les liaisons entre ville-nature ${ }^{1}$ et architecture des milieux ${ }^{2}$, centrées sur les questions d'habiter " et sur celles de lieux et de milieux ». Le milieu ne va pas sans multiplicité ; il y a toujours plusieurs milieux en jeu (social, naturel, technique, culturel, psychologique...), mais ceux-ci se superposent et s'entremêlent. De façon concomitante, tout est déjà là, et en même temps tout advient, sans qu'on puisse rendre compte d'un commencement ni d'une fin, comme dans la figure rhizomatique. "On commence toujours au milieu ", dit Deleuze et c'est à travers des milieux que nous accédons au monde. Un monde qui ne se situe pas au-delà des milieux, comme à un étage supérieur mais dans leur rencontre.

\section{Ville-nature / ville-monde}

Les milieux font monde. Welt et Umwelt ne se distinguent pas comme deux régions hétérogènes, et c'est leurs différences subtiles et leurs articulations qu'il s'agit d'envisager. C'est parce qu'il y a

1. Chris Younès (dir.), Ville

contre-nature, Paris, La Découverte, 1999.

2. C. Younès, Benoît Goetz (dir.),

"L'architecture des Milieux », Le Portique, 2010. 
des milieux et non un seul que nous pouvons avoir un monde, mais cela n'est pas donné de droit. Cela doit être construit à différents niveaux. Dans Les trois écologies ${ }^{3}$, Félix Guattari définit l'écosophie comme "I'écologie environnementale d'un seul tenant avec l'écologie sociale et mentale, à travers une écosphère de caractère éthico-politique ", mettant l'accent sur les territoires existentiels, les pluralités, les hétérogenèses, les perspectives éthiques et politiques, les singularités, les nouvelles pratiques esthétiques... c'est-à-dire sur ce qui fait évènement tout en restant indissociable de ce qui fait monde. L'écologie, science qui étudie les milieux des différents êtres vivants, met en évidence à la fois comment les milieux déterminent leurs vies, et comment ces êtres interagissent avec les milieux. Ainsi l'écologie est la fille de la mésologie ( science des milieux ») et la sœur de l'éthologie (" biologie dynamique »)4. L'architecture des milieux relève de ces trois disciplines. Elle concerne aussi bien la physique (donc, littéralement, la nature) des établissements humains que la politique, indissociable de la condition urbaine, la ville étant le milieu humain par excellence. Ce qui rend les villes respirables (ou pas) c'est certes la qualité de l'air mais aussi la manière dont les rues et les places le font circuler, la manière de vivre ensemble quelque part. Ce qu'on appelle parfois la "ville générique » n'a pas absorbé la variété infinie des différences entre les villes et dans les villes.

Tout milieu singulier est déjà en lui-même une multiplicité. La célébrité de la tique de Jacob von Uexküll ${ }^{5}$ tient à ce que l'on parvient avec elle à isoler aisément les composantes d'un milieu très simple (mais pourtant déjà pluriel) ; tandis que les milieux humains comportent d'innombrables composantes. Les mondes humains sont-ils alors essentiellement différents des milieux animaux? La question peut se poser mais après l'étude approfondie et patiente de la notion de milieu.

Si on peut parler de ville-nature, c'est en un sens qui chamboule profondément notre idée de la nature et du « naturel ». II ne faut pas être trop naïf quand on aborde cette question de la ville-nature (en la confondant, par exemple, avec la «végétalisation » devenue une mode). La ville-nature n'est pas une ville qui serait " naturelle » en tant que telle, mais une ville qui renouerait avec les éléments de manière inédite. En premier lieu avec la Terre. La ville est déjà devenue une « nature » et cela au sens littéral et simple de l'expression, en accueillant la vie de beaucoup d'animaux, parce qu'elle est souvent plus hospitalière que les « campagnes » dévastées. Les "villes-nature " sont donc les enjeux d'écologies urbaines complexes qui activent une pensée des milieux. L'enjeu est celui de la vie, dans toutes les acceptions de ce terme qui ne sont pas seulement biologiques. Mais il est indéniable que le vivant - le bios - fournit aujourd'hui un nouveau paradigme. "II n'y a pas "le" milieu mais le milieu "de" " explique Georges Canguilhem en soulignant les liens étroits entre le vivant et le milieu : "Enlevez le milieu, vous enlevez le vivant. Si vous sortez un poisson de l'eau, il sera mort. "Nous sommes ainsi invités à penser en termes d'interdépendance, de totalité et d'évolutivité, d'unités composites d'échanges, d'interactions, alors même que trop souvent des logiques technocratiques, disciplinaires, économiques, séparent, objectivent voire instrumentalisent.
3. Félix Guattari, Les trois écologies, Paris, Galilée coll. «L'espace critique », 1989.

4. Dominique Ottavi, " Mésologie », Bulletin de la Société française pour I'histoire des sciences de I'homme, $\mathrm{n}^{\circ}$ 32, hiver 2008, p. 48-49.
5. Uexküll a analysé comment le monde

d'un animal fait intervenir perception et conduite, et comment apparaît un milieu d'événements ouvrant un champ spatiotemporel. Jacob von Uexküll, Mondes animaux, monde humain, Paris, Gonthier, 1956. 
Georg Gerster, Le Pain et Le Sel, Paris, Arthaud, 1980.

Photographie aérienne, province de Kermân, Iran. Dans cet ouvrage, Gerster insiste sur la fragilité des milieux qu'il photographie.

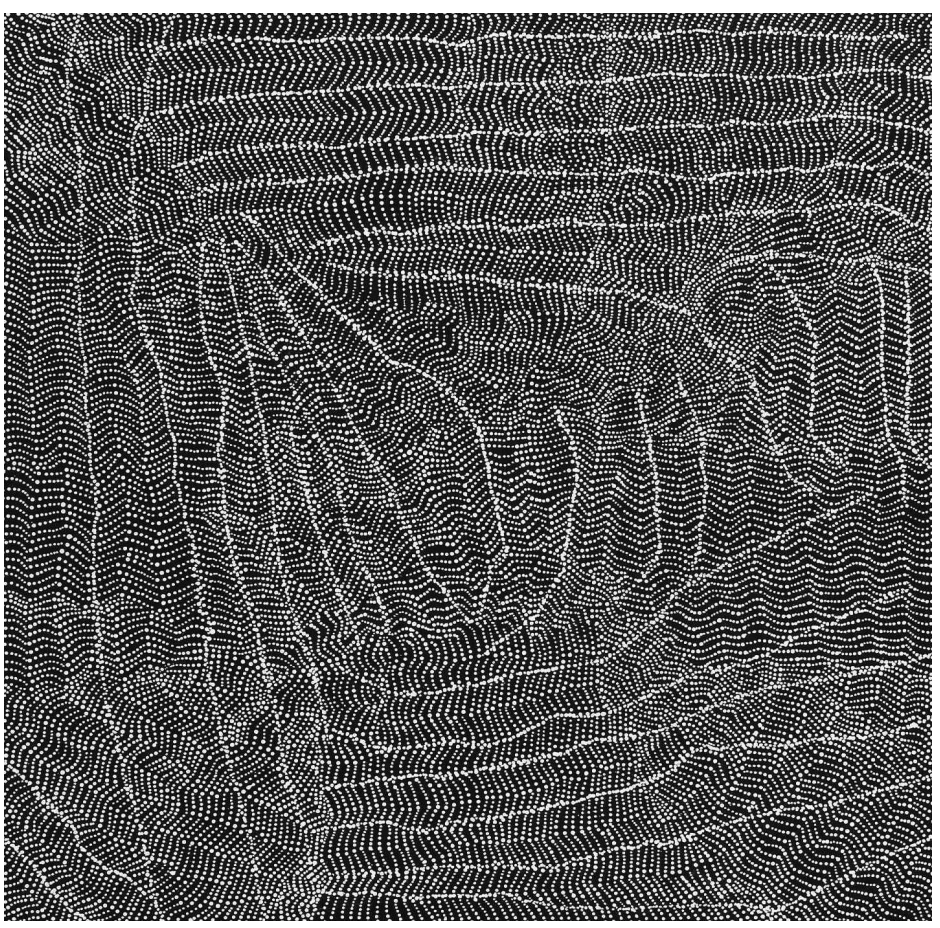

Dorothy Napangardi,

Huile sur toile, 2008.

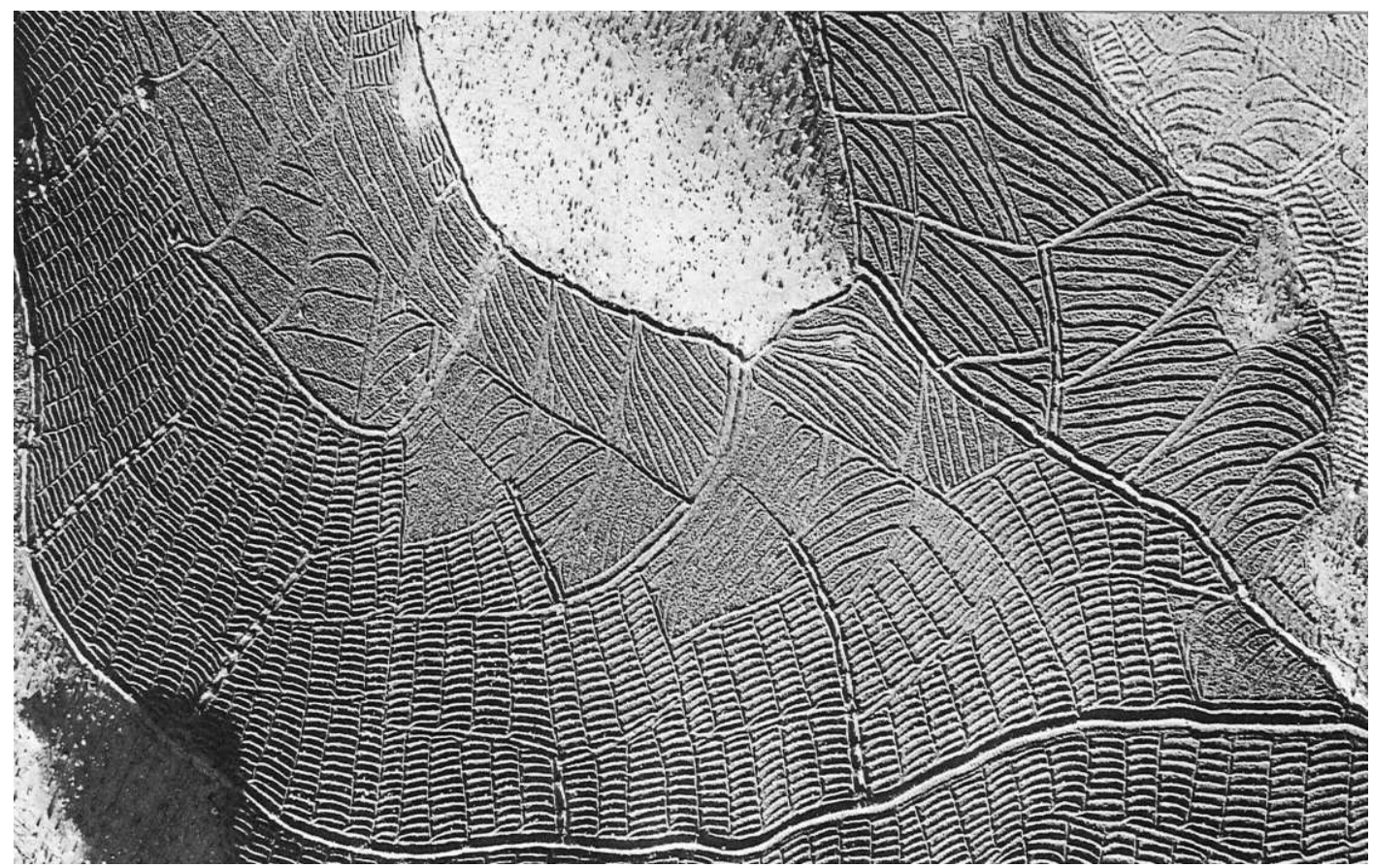




\section{Vers un paradigme de la reliance}

C'est donc en tant que changement paradigmatique privilégiant des dispositifs de relations, que s'explorent, dans le contexte du "développement durable ", I'invention d'une ville-nature et d'une architecture des milieux. En effet, un milieu - lieu ou biotope - requière de comprendre les interactions et les dynamiques naturo-culturelles d'interpénétrations et d'interdépendances, que ce soit entre facteurs climatiques, mécaniques et chimiques, biotiques ou culturels. Mais dès que l'attention est portée sur un habiter spécifique, à savoir sur la façon d'être au milieu, c'est en termes de relations qu'il peut être décrit. Des opérateurs anthropo-architecturaux spatio-temporels permettent de caractériser plus particulièrement ce qui met en synergie la partie et le tout : la limite qui distingue, l'espacement ou écart qui met à distance, coupe, sépare tout en ménageant une certaine proximité, mais aussi les passages et porosités entre les choses et les êtres. Face à une certaine modernité qui a privilégié la séparation et la maîtrise, c'est à une autre écologie de I'action que nous sommes conviés. Edgar Morin a fait du concept de reliance ${ }^{6}$ la cellule souche de la pensée complexe, comme "le travail des liens », "l'acte de relier et de se relier et son résultat ". II s'agit d'un changement paradigmatique permettant d'optimiser à la fois les rapports de l'anthropisation au milieu naturel et les conditions du vivre-ensemble. En architecture, il apparaît désormais à nouveau nécessaire de savoir s'ajuster aux contextes, de s'attacher aux situations locales avec leurs spécificités plutôt que de procéder à une tabula rasa ou d'en rester à des modèles génériques ou des recettes préétablies, incapables de prendre en compte les conditions d'implantation d'un projet.

\section{Coexistences}

L'enjeu majeur désormais est de penser et imaginer les conditions soutenables d'une vie sur terre qui menace d'épuisement. II y a une grande fécondité de la redéfinition des reliances ville-nature qui créent des conditions $d$ 'habiter alternatives et contribuent à une refondation réelle, imaginaire et symbolique de lieux urbains en symbiose avec le vivant. Ce mixte entre urbain et nature s'exprime sous plusieurs configurations : densité raisonnée préservant des espaces non bâtis de forêt, de campagne et de nature sauvage, mails plantés, jardins et parc urbains, création de microclimats... II est privilégié de travailler avec l'eau, le vent, le soleil, le sol, la faune, la flore, de recycler, réemployer, hériter, économiser les ressources, établir des solidarités, se déplacer suivant des modes diversifiés de mobilité qui permettent de lutter contre la pollution et les formes de ségrégation socio-spatiales. Concevoir de tels milieux habités requiert des coexistences, équilibres, adaptations, réinventions et re-créations entre natures et artifices, cultures urbaines et agricoles, écosystèmes et anthropisation. En fait, ce sont les résiliences comme les ré-enchantements qui se retrouvent au cœur d'une architecture des milieux.

\section{Invention architecturale et transmilieu(x ${ }^{7}$}

Les multiples enjeux que soulève la question de la ville-nature, rappelés ci-dessus, invitent à réévaluer de fond en comble les modalités de l'invention architecturale. En effet, derrière la force de l'urgence qui
6. Morin expose cette pensée de la reliance dans La méthode, qui se présente sous la forme de six tomes, mais elle est plus particulièrement explicitée et revendiquée dans le dernier, intitulé "Éthique ». II considère que la notion de reliance inventée par le sociologue Marcel Bolle de Bal, comble un vide conceptuel en donnant une nature substantive à ce qui n'était conçu qu'adjectivement et en donnant un caractère actif à ce substantif. Edgar Morin, La méthode 6. Éthique, Paris, Le Seuil, 2004.
7. Cette problématique est développée dans la thèse de doctorat de philosophie de Stéphane Bonzani, La ligne d'édifier. Invention architecturale et transmilieu(x), soutenue le 22 septembre 2010. 
est souvent impliquée dans "l'impératif écologique", une transformation en sous-œuvre s'opère quant à la façon dont l'architecte conçoit et définit sa propre pratique créatrice. "Qu'est-ce que je fais au juste quand je fais, ou prétends faire de la philosophie ?" s'interrogeait Gilles Deleuze dans une conférence ${ }^{8}$. Nous aimerions ici déplacer cette question vers l'architecture. En quoi consiste au juste l'invention architecturale?

De même que la création de concepts ne suffit pas à définir l'activité d'un philosophe, la création d'édifices ne suffit pas à rendre compte de celle d'un architecte. L'importance accordée par Deleuze et Guattari à l'instauration d'un plan d'immanence comme autre face de l'invention philosophique est à transposer dans l'invention architecturale. Cette activité en sous-œuvre n'est pas secondaire. Elle ne précède pas la création de concept, ni ne lui succède. Elle est simultanée et déterminante mais ne se donne jamais pour elle-même, si bien qu'elle passe le plus souvent inaperçue.

\section{Instaurer des milieux}

Le problème de l'instauration n'est pas le même que celui de la création, bien qu'il y soit impliqué et que toute création engage une instauration. C'est à Étienne Souriau que l'on doit la première tentative d'explicitation de cette opération complexe ${ }^{9}$. Cherchant à saisir le mode d'existence de l'œuvre à faire ${ }^{10}$, Souriau convoque l'instauration pour désigner le passage ou le trajet de l'ébauche à l'œuvre accomplie. Dans un autre registre, Gilbert Simondon se confronte à une investigation portant sur la genèse de l'objet technique ${ }^{11}$. Pour lui, l'émergence d'un tel objet, qu'il nomme processus de concrétisation, se double nécessairement de la mise en place d'un " milieu associé ", condition de fonctionnement de l'objet et milieu hybride entre l'environnement propre à l'objet technique et le milieu géographique ou naturel. De même que le plan d'immanence n'est pas créé, mais instauré, le milieu associé «n'est pas fabriqué, ou tout du moins pas fabriqué en totalité ».

Invention d'une œuvre d'art, d'un concept philosophique, d'un objet technique partagent, selon ces penseurs, une même problématique : la constitution de milieux qui ne s'identifient pas avec les objets créés mais en tracent les conditions nécessaires d'existence. Or ces milieux, qui sont des entités bien étranges, possèdent quelques propriétés communes : ils sont constitués pendant (et non avant ou après) l'invention elle-même, ils sont générés par l'invention bien qu'ils en forment les conditions, ils se présentent comme des interfaces entre l'objet de la création et un fond qui en forme le dehors, ils sont donc toujours ouverts sur une extériorité, ils sont hétérogènes par nature et tissent ensemble des morceaux ou découpages de réalités. Nous sommes ici très proches de la conception du milieu de Uexküll, qui introduit le Umwelt comme un espace découpé sur le Umgebung c'est-à-dire l'espace géographique banal. Ce milieu est la configuration d'un espace-temps signifiant pour un vivant, constituant un champ signifiant pour lui.

Quel est le milieu instauré par l'invention architecturale ? L'architecture est indissociablement politique et technique. Le milieu qu'elle instaure vise deux objectifs complémentaires : tisser des liens entre les hommes et tisser des liens entre cette société et la nature qui la supporte. Cette double ambition n'est évidemment
8. "Qu'est-ce que l'acte de création?», conférence donnée à la FEMIS, école nationale supérieure des métiers de l'image et du son. Gilles Deleuze, Deux régimes de fous, Paris, Minuit, 2003, p. 291-302.

9. Étienne Souriau, L'instauration philosophique, Paris, Éditions Alcan, 1939.
10. É. Souriau, Du mode d'existence de l'œuvre à faire, extrait du Bulletin de la Société française de philosophie, 50 (1), séance du 25 février 1956, p. 4-24.

11. Gilbert Simondon, Du mode d'existence des objets techniques, Paris, Aubier, 1958. 
pas nouvelle et une lecture attentive des textes qui ont cherché à définir cet art le confirme. C'est sans doute Alberti qui signe avec le plus de force cette articulation profonde entre la constitution d'un monde humain et la construction des dispositifs techniques qui composent avec des forces naturelles. Chez cet humaniste inquiet, l'activité édificatrice est la condition sine qua non de l'institution d'une solidarité entre les membres d'une société en devenir ${ }^{12}$, résistance stoïcienne face à la fortuna. Mais déjà chez Vitruve ${ }^{13}$, on comprend que la difficulté de l'architecture sera de maintenir ensemble des forces très diverses, comme en témoigne la section du De architectura concernant l'édification d'une ville, au croisement des vents, du soleil, des dieux et du commerce des hommes ; ou encore le vertigineux passage consacré aux six exigences auxquelles doit répondre la conception d'un édifice, où l'on traverse des strates telles que la proportion, l'usage, ou le décor, chacune mobilisant des domaines distincts. Les nombreuses définitions ${ }^{14}$ qui ponctuent I'histoire de l'architecture insistent toutes sur cette synthèse de l'hétérogène par l'archè. Eurythmie, harmonie, convenance, composition, assemblage, cohérence, autant de termes distillés au cours des textes visant à expliciter cette articulation complexe.

L'architecture est donc art de la synthèse. Certes, mais le problème serait simple si les composantes à agencer (hommes, éléments naturels, animaux, végétaux, lumière, vents, etc.) se présentaient libres, déliées, atomes offerts à de savants agencements. Ce n'est évidemment pas le cas. Ces composantes sont elles-mêmes prises dans des milieux. Si bien que faire un édifice, ou une ville, disons plus généralement un dispositif architectural, consiste, «par le déplacement des masses, par la liaison et par l'assemblage des $\operatorname{corps}^{15}$ " à mettre des milieux en rapport, en synergie. II s'agit donc d'instaurer ce que nous proposons d'appeler un transmilieu(x). Par là, nous entendons moins un ensemble fini et clos sur lui même qu'une ouverture (Maldiney). Nous n'entendons pas un champ stabilisé mais une variation, un mouvement (Focillon ${ }^{16}$ ). Nous n'entendons pas non plus une instance englobante, une sorte de métamilieu qui viendrait rassembler sous sa coupe la diversité des milieux qu'il convoque, mais un passage entre des milieux qui, par lui, deviennent autres, raison pour laquelle l'opération constitutive de transmilieu( $x)$ n'est pas une simple combinaison mais une authentique instauration qui ne peut être que rythmique.

Définir l'invention architecturale comme l'instauration rythmique de transmilieu(x) s'inscrit évidemment dans un contexte géographique et historique précis. Le contexte géographique, c'est celui d'une planète saturée des traces humaines, d'un écoumène faisant corps avec la Terre, pour la première fois dans I'histoire humaine, rendant l'expansion impossible et invitant à réfléchir à des stratégies de régénération des milieux habités. Le contexte historique, c'est celui d'un héritage que la modernité nous a légué, inscrivant l'art d'édifier dans une ignorance des milieux et des rythmes.

\section{De la figure au motif}

De cet entrelacement d'interdépendances et de temporalités ne peut résulter ni système formel, ni règles préétablies; règles et systèmes que la modernité, en forme de rupture après des siècles de recherche de
12. Leon Battista Alberti, De re aedificatoria, 1485, trad. fr. Françoise Choay, Pierre Caye, L'art d'édifier, Paris, Le Seuil, 2003.

13. Vitruve, De architectura, trad. fr. Claude Perrault, Les dix livres d'Architecture, 1684.

14. Benoit Goetz, Philippe Madec, Chris Younès, L'indéfinition de I'architecture. Un appel, Paris, Éditions de la Villette, 2009.
15. L. B. Alberti, De re aedificatoria, op. cit. note 13, p. 48 .

16. Henri Focillon, Vie des formes, Paris, PUF, p. 95 : «La notion de milieu ne doit [...] pas être acceptée à l'état brut. II faut la décomposer, reconnaître qu'elle est une variable, un mouvement $»$. 
l'équilibre absolu en architecture, avait paradoxalement cherché à fixer à nouveau. C'est bien pourtant un changement de paradigme esthétique qui est ici en jeu. II n'est plus question de proposer des combinaisons d'éléments ou des dispositifs composés, mais de renouveler les conditions de l'émergence des transformations, en modifiant les concepts architecturaux de référence. Quelles pistes explorer?

Première piste, l'abandon du cadre et, par conséquent, la mutation radicale du rapport de l'objet au site, du " contexte » et des externalités de l'objet architectural. Le sol n'est plus ce qui porte l'architecture, une condition préalable, dont les dimensions différentes, emboîtées comme des échelles gigognes, en constitueraient successivement l'environnement: I'assise, la parcelle, le voisinage, la région. L'architecture des milieux - et le concept de transmilieu $(x)$ - remettent en cause la figure singulière de l'architecture sur " son " site. Ils interrogent le «zoom » et la séparation des savoirs qui lui est associée - séparant architecture, urbanisme et géographie ${ }^{17}$. Ce qui est construit peut conserver ses propres limites, son propre temps d'édification, mais ne se conçoit pas comme un phénomène isolé, ne serait-ce qu'en vertu des forces naturelles qui l'englobent et avec lesquelles il coexiste, des continuités des modes de production et des expériences sensibles (l'architecture comme expérience ne s'arrête pas aux seuils de l'édifice et se prolonge vers les milieux associés). La dimension esthétique de cette nouvelle condition fait écho à la diversité de la production artistique des dernières décennies, où l'objet n'est plus au « milieu » de l'œuvre, et cède le pas au mouvement, au devenir, au processus ou à la vie elle-même, dans toutes ses dimensions, physiques, interactives, combinatoires, inachevées, imprévisibles, destructrices même ${ }^{18}$. Ce renouvellement trouve ses sources dans la modernité, au début du xx siècle. Chez Matisse, par exemple, qui interprète l'énergie vitaliste des fauves, et s'attache bien plus qu'au hors cadre à faire en sorte que la peinture impacte profondément son environnement, le lieu où elle s'inscrit, où elle se fabrique, et devienne un véritable décor (" dans lequel la vie même a lieu au quotidien») dynamique et afocal ${ }^{19}$. Des êtres vivants, le peintre désignait leur forme comme étant «le dessin $d^{\prime}$ un mouvement ${ }^{20}$ ». De la même manière, les énergies naturelles en jeu mêlent à toutes les échelles les socles communs (le sol, le climat), les liens plus étendus et les interstices resserrés, entremêlent continuités et discontinuités spatiales et temporelles, pour former, bien plus qu'une série de figures juxtaposées, un motif contrasté, variable, à la fois unitaire et diversifié - un peu comme on le dirait d'un tissage, dont les nuances n'altèrent pas la cohérence globale, et en son étroitement liées, et participent de la fabrique de l'ensemble. Tout se tient, et pourtant tout varie.

Deuxième piste, l'importance de la fabrication, le lien des variations formelles, des mesures et des écritures avec les modes opératoires. Plus que le résultat, la manière de faire, la négociation entre le geste et la matière confère une dimension esthétique au milieu transformé, le liant dans le même temps à ses conditions historiques, sociales et économiques. Pour compléter la lecture d'Alberti, relisons l'œuvre clé de Gottfried Semper ${ }^{21}$, s'éloignant de la quête de l'optimi-
17. Dans le livre Milieux conçu par

Patrick Berger, Jade Lindgaard parle de

"dimension conjointe » entre architecture et urbanisme (p. 36). Xavier Douroux suggère que les hypothèses de Berger font écho à "l'échec de l'urbanisme» (p. 149). Patrick Berger, Milieux, Paris, Édition Cité de I'architecture et du patrimoine, catalogue de l'exposition éponyme, 2005.
18. John Dewey, Art as Expérience, New-York, Perigee Books,1934 ; trad. française, L'art comme expérience, Paris, Gallimard coll. «Folio Essais », 2010.

19. Éric Alliez, Jean-Claude Bonne, La pensée Matisse, portrait de l'artiste en hyper fauve, Paris, Le Passage, 2005.

20. É. Alliez, J.-C. Bonne, op. cit. note 19, p. 57. 


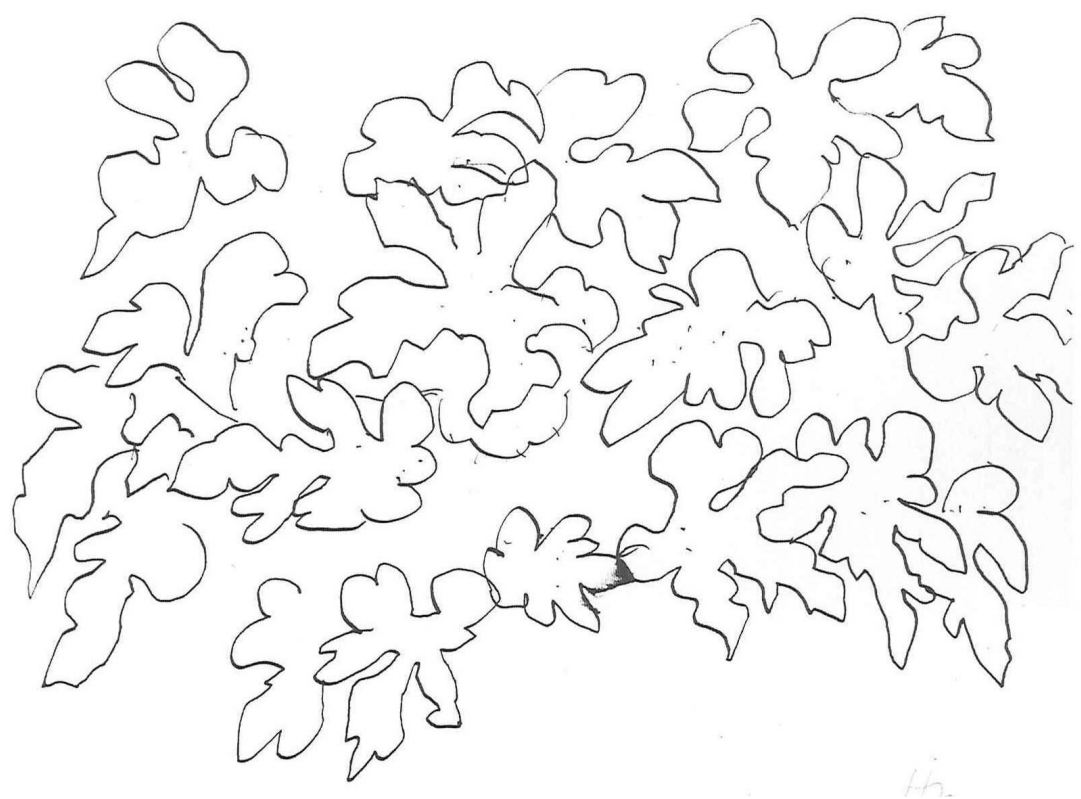

Henri Matisse, Feuilles de figuier, ca. 1941. Extrait de l'ouvrage La Pensée-Matisse. Portrait de l'artiste en hyperfauve, Éric Allier et Jean-Claude Bonne, Paris, Le Passage, 2005.

sation géométrique des textes antérieurs ${ }^{22}$ pour inscrire I'esthétique architecturale dans I'héritage vernaculaire des techniques ${ }^{23}$. Semper suit I'héritage des Lumières, et tente l'équivalent architectural des synthèses d'un Newton pour la physique des corps ou d'un Humboldt pour le vivant et les milieux, englobant tous les temps connus et toutes les géographies explorées. Mère des techniques, le textile y prend la place ignorée par les textes antérieurs. Quatre techniques élémentaires - le tissage, la céramique, la charpente et la maçonnerie, qui correspondent à quatre états de la matière la fibre souple, la plasticité, la fibre rigide, la masse tectonique; quatre potentiels sans cesse en devenir, et non plus seulement des figures déchiffrées et stabilisées, mais une manière de lier nature et technique. Ce texte est une source encore mal explorée pour enrichir une esthétique où deux écritures s'entrelacent dans un même motif. Celle, interne, des forces de la matière devenue structure ou édifice, celle, externe, du sol remodelé par cette installation nouvelle ou des dynamiques naturelles du vivant ${ }^{24}$.

Troisième piste, l'interopérabilité et l'interactivité fortement liées aux nouvelles conditions sociales de la production architecturale. Comment penser l'œuvre
21. Gottfried Semper, Style in the Technical and Tectonics Arts or, Practical Aesthetics, Los Angeles, Getty Research Institute, 1993, trad. anglaise par Harry Francis Mallgrave et Michael Robinson du texte original paru à Zürich en 1860

\section{Semper désignant par exemple} les travaux de Durand comme un "schématisme sans vie ", et ceux de Rondelet comme "purement pratiques». H. F. Mallgrave, op. cit. p. 12.
23. Au sens tel qu'éclairé par John Brinckerhoff Jackson.
24. On retrouve dans cette tension entre forces externes et internes aussi bien I'hypothèse d'Éric Alliez et Jean-Claude Bonne sur les motifs de Matisse et leurs liens entre ornementalité (dispositif interne) et décoratif (récit externe) - La pensée Matisse, op. cit. note 19, p. 67 et p. 70 - que celle développée par Xavier Douroux à propos du travail de Patrick Berger, entre pression interne de la "structure » et pression externe du " site », Milieux, op. cit. note 17, p. 153. 
190 Les Cahiers de la recherche architecturale et urbaine - n²6/27

Trajectoires doctorales

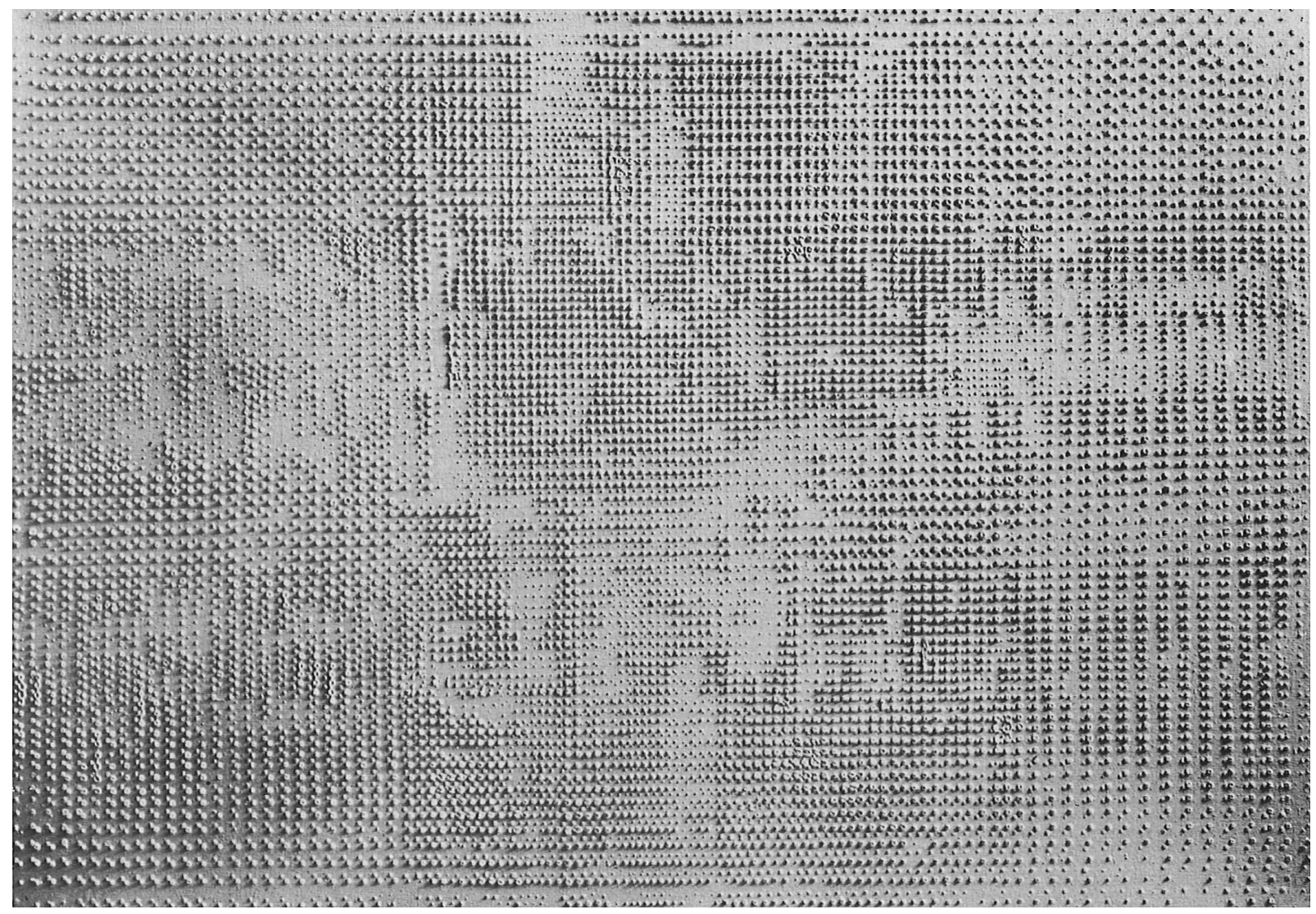

Otto Piene, peinture sur toile, 1960.

Illustration d'un des livres de Gyorgy

Kepes, La structure dans les arts et

dans les sciences, 1967, Bruxelles,

La Connaissance (Bibliothèque de

synthèses). Ouvrage cité par Patrick

Berger et Xavier Douroux dans

le livre Milieux, Institut français

d'architecture, 2005. 
esthétique à l'aune de la participation citoyenne, du débat contradictoire, de l'instabilité programmatique, et de sa reconversion prochaine, toujours plus ou moins sous-jacentes, qu'il s'agisse des transformations induites par l'usage ou par l'économie ou de son recyclage ? De manière analogue, quelle qu'en soit l'écriture, l'architecture pensée comme système ouvert, peut, sans être indéterminé, "offrir des possibilités, devenir espace d'émancipation, de transgression même ${ }^{25}$ ». Le paradigme esthétique de l'œuvre définitive, suspendue dans le temps par une équation absolue, ne tient plus ici. Soit l'on cède au bricolage et à l'approximation successive (stimulante perspective, d'ailleurs) soit l'on cherche, sous forme de matrices évolutives, un modèle qui soit à la fois une géométrie et une offre illimitée de variations, sans qu'en pâtisse la cohérence. Tentative qui fut celle de Alvar Aalto, qui se référait aux sciences naturelles et à la puissance de renaissance de la nature, capable d'induire sans jamais les répéter le même arbre, branche ou feuillage; de cette " architecture précise pour toute forme de vie ${ }^{26}$ " émergent de nouveaux motifs, un autre imaginaire. II y a une part de jeu dans cette esthétique matricielle, support actif d'une architecture des milieux.

Quatrième piste, le mobile comme représentation dynamique des équilibres, d'une économie générale des milieux et des interdépendances entre leurs parties. Le déplacement de matières que toute transformation du milieu suppose ne peut advenir innocemment, sans élaborer la conscience de l'équilibre des ressources et de leur finitude, en alliant ici politique et esthétique. Ce qui advient ici intervient

25. P. Berger, op. cit. note 17 ; texte de Wouter Davidts et Xavier Douroux, p. 38.

26. P. Berger, op. cit. note 17, « les figures d'espaces et les valeurs d'usage ». sur un ailleurs. Cette influence distante interroge à la fois l'idée de bien commun et celle de juste mesure. Le partage du sol, la part de l'espace public et des dispositifs de séparation, par exemple, pourraient susciter de nouveaux agencements, plus enclins à recréer reliances et passages.

Ces quatre pistes ne constituent pas des réponses, mais plutôt des hypothèses pour transformer l'univers esthétique architectural.

Plutôt que la figure, repérable par contraste, selon un mode de composition cadré dans l'espace et dans le temps, c'est le motif, plus indéterminé, soumis à de multiples variations, qui correspond le mieux à cette nouvelle condition. Ce qui est construit et transformé émerge, en quelque sorte, d'un fond dont on ne peut complètement le dissocier, qui le porte et qu'il constitue à la fois. 